\title{
Singleton sets random attractor for stochastic FitzHugh-Nagumo lattice equations driven by fractional Brownian motions 1
}

\author{
Anhui Gu, Yangrong Li \\ School of Mathematics and Statistics, Southwest University, Chongqing 400715, China
}

\begin{abstract}
The paper is devoted to the study of the dynamical behavior of the solutions of stochastic FitzHugh-Nagumo lattice equations, driven by fractional Brownian motions, with Hurst parameter greater than $1 / 2$. Under some usual dissipativity conditions, the system considered here features different dynamics from the same one perturbed by Brownian motion. In our case, the random dynamical system has a unique random equilibrium, which constitutes a singleton sets random attractor.
\end{abstract}

Keywords: Stochastic FitzHugh-Nagumo lattice equations; fractional Brownian motion; random dynamical systems; random attractor.

\section{Introduction}

Recently, the dynamics of deterministic lattice dynamical systems have drawn much attention of mathematicians and physicists, see e.g. [1]-[5] and the references therein. As we know, most of the realistic systems involve noises which may play an important role as intrinsic phenomena rather than just compensation of defects in deterministic models. Stochastic lattice dynamical systems (SLDS) arise naturally while these random influences or uncertainties are taken into account. Since Bates et al. [6] initiated the study of SLDS, many works have been done regarding the existence of global random attractors for SLDS with white noises on infinite lattices (see e.g. [7]-[11]). Later, the existence of global random attractors was extended to other SLDS with additive white noises, for example, first-order SLDS on $\mathbb{Z}^{k}[$ ] , stochastic Ginzburg-Landau lattice equations [9], stochastic FitzHugh-Nagumo lattice equations [10], second-order stochastic lattice systems [11] and first (or second)-order SLDS with a multiplicative white noise [7, 12]. Zhao and Zhou [13] gave some sufficient conditions for the existence of a global random attractor for general SLDS in the non-weighted space $\mathbb{R}$ of infinite sequences and provided an application to damped sine-Gordon lattice systems with additive noises. Very recently, Han et al. [14] provided some sufficient conditions for the existence of global compact random attractors for

\footnotetext{
${ }^{1}$ This work has been partially supported by NSFC Grants 11071199, NSF of Guangxi Grants 2013GXNSFBA019008 and Guangxi Provincial Department of Research Project Grants 2013YB102.
} 
general SLDS in the weighted space $\ell_{\rho}^{p}(p \geqslant 1)$ of infinite sequences, and their results are applied to second-order SLDS in [15] and [16].

However, as can be seen that all the work above are considered in the framework of the classical Itö theory of Brownian motion. Note that fractional Brownian motion ( $\mathrm{fBm}$ ) does not possess independent increments and stochastic differential equations driven by fBm do not define the Markov process as in the case of usual white noises. Therefore, it is not possible to apply standard methods (see e.g. Theorem 3.1 in [14]) to deal with these questions. Fortunately, the theory of random dynamical systems still works for non-Markovian processes (see [17, 18]). In [19], we consider the first-order lattice dynamical system perturbed by fractional Brownian motions.

FBm appears naturally in the modeling of many complex phenomena in applications when the systems are subject to "rough" external forcing. An fBm is a stochastic process which differs significantly from the standard Brownian motion and semi-martingales, and other classically used processes in the theory of stochastic process. As a centered Gaussian process, it is characterized by the stationarity of its increments and a medium- or long-memory property. It also exhibits power scaling with exponent $H$. Its paths are Hölder continuous of any order $H^{\prime} \in(0, H)$. An $\mathrm{fBm}$ is not a semi-martingale nor a Markov process. Especially, when the Hurst parameter $H \in(1 / 2,1)$, the $\mathrm{fBm}$ has the properties of self-similarity and long-range dependence. So, $\mathrm{fBm}$ is the good candidate to model random long term influences in climate systems, hydrology, medicine and physical phenomena. For more details on fBm, we can refer to the monographs [20, 21].

Motivated by [10, 19], we investigate the long-term behavior of the following stochastic FitzHugh-Nagumo lattice equations:

$$
\left\{\begin{array}{l}
\frac{d u_{i}}{d t}=u_{i-1}-2 u_{i}+u_{i+1}-\lambda u_{i}+f_{i}\left(u_{i}\right)-v_{i}+a_{i} \frac{d \beta_{i}^{H}(t)}{d t}, \\
\frac{d v_{i}}{d t}=\varrho u_{i}-\sigma v_{i}+b_{i} \frac{d \beta_{i}^{H}(t)}{d t}, \\
u(0)=u_{0}=\left(u_{i 0}\right)_{i \in \mathbb{Z}}, \quad v(0)=v_{0}=\left(v_{i 0}\right)_{i \in \mathbb{Z}},
\end{array}\right.
$$

where $\mathbb{Z}$ denotes the integer set, $u_{i} \in \mathbb{R}, \lambda, \varrho$ and $\sigma$ are positive constants, $f_{i}$ are smooth functions satisfying some dissipative conditions, $\left(a_{i}\right)_{i \in \mathbb{Z}} \in \ell^{2},\left(b_{i}\right)_{i \in \mathbb{Z}} \in \ell^{2}$ and $\left\{\beta_{i}^{H}: i \in \mathbb{Z}\right\}$ are independent two-sided fractional Brownian motions with Hurst parameter $H \in(1 / 2,1)$, $\ell^{2}=\left(\ell^{2},(\cdot, \cdot),\|\cdot\|\right)$ denotes the regular space of infinite sequences. When there are no noises terms, form similar to (1.1) is the discrete of the FitzHugh-Nagumo system which arose as modeling the signal transmission across axons in neurobiology (see [22]). FitzHugh-Nagumo lattice system was used to stimulate the propagation of action potentials in myelinated nerve axons (see [23]). The stochastic FitzHugh-Nagumo lattice equations were first proposed in [10]. The existence of random attractors of (similar) stochastic FitzHugh-Nagumo lattice equations 
with white noises were established in [10, 24] and [25].

The goal of this article is to establish the existence of a random attractor for stochastic FitzHugh-Nagumo lattice equations with the nonlinear $f$ under some dissipative conditions and driven by fractional Brownian motions with Hurst parameter $H \in(1 / 2,1)$. By borrowing the main ideas of [26], we first define a random dynamical system by using a pathwise interpretation of the stochastic integral with respect to the fractional Brownian motions. This method is based on the fact that a stochastic integral with respect to an $\mathrm{fBm}$ with Hurst parameter $H \in(1 / 2,1)$ can be defined by a generalized pathwise Riemann-Stieltjes integral (see e.g. [27]-[30]). And then we show the existence of a pullback absorbing set for the random dynamical system achieved by means of a fractional Ornstein-Uhlenbeck transformation and Gronwall lemma. Since every trajectory of the solutions of system (1.1) cannot be differentiated, we have to consider the difference between any two solutions among them, which is pathwise differentiable (see [26]). Due to the stationarity of the fractional Ornstein-Uhlenbeck solution, we get a unique random equilibrium finally. All solutions converge pathwise to each other, so the random attractor, which consists of a unique random equilibrium, is proven to be a singleton sets random attractor.

The paper is organized as follows. In Sec. 2, we recall some basic concepts on random dynamical systems. In Sec. 3, we give a unique solution to system (1.1) and make sure that the solution generates a random dynamical system. We establish the main result, that is, the random dynamical system generated by equation (1.1) has a unique random equilibrium, which constitutes a singleton sets random attractor in Sec. 4.

\section{Preliminaries}

In this section, we introduce some basic concepts related to random dynamical systems and random attractors, which are taken from [31]-33].

Let $\left(\mathbb{E},\|\cdot\|_{\mathbb{E}}\right)$ be a separable Hilbert space and $(\Omega, \mathcal{F}, \mathbb{P})$ be a probability space.

Definition 2.1. A metric dynamical system $(\Omega, \mathcal{F}, \mathbb{P}, \theta)$ with two-sided continuous time $\mathbb{R}$ consists of a measurable flow

$$
\theta:(\mathbb{R} \times \Omega, \mathcal{B}(\mathbb{R}) \otimes \mathcal{F}) \rightarrow(\Omega, \mathcal{F}),
$$

where the flow property for the mapping $\theta$ holds for the partial mappings $\theta_{t}=\theta(t, \cdot)$ :

$$
\theta_{t} \circ \theta_{s}=\theta_{t} \theta_{s}=\theta_{t+s}, \quad \theta_{0}=\operatorname{id}_{\Omega}
$$

for all $s, t \in \mathbb{R}$, and $\theta \mathbb{P}=\mathbb{P}$ for all $t \in \mathbb{R}$. 
Definition 2.2. A continuous random dynamical system $(R D S) \varphi$ on $\mathbb{E}$ over $\left(\Omega, \mathcal{F}, \mathbb{P},\left(\theta_{t}\right)_{t \in \mathbb{R}}\right)$ is a $\left(\mathcal{B}\left(\mathbb{R}^{+}\right) \times \mathcal{F} \times \mathcal{B}(\mathbb{E}), \mathcal{B}(\mathbb{E})\right)$-measurable mapping and satisfies

(i) $\varphi(0, \omega)$ is the identity on $\mathbb{E}$;

(ii) $\varphi(t+s, \omega)=\varphi\left(t, \theta_{s} \omega\right) \circ \varphi(s, \omega)$ for all $s, t \in \mathbb{R}^{+}, \omega \in \Omega$;

(iii) $\varphi(t, \omega)$ is continuous on $\mathbb{E}$ for all $(t, \omega) \in \mathbb{R}^{+} \times \Omega$.

A universe $\mathcal{D}=\{D(\omega), \omega \in \Omega\}$ is a collection of nonempty subsets $D(\omega)$ of $\mathbb{E}$ satisfying the following inclusion property: if $D \in \mathcal{D}$ and $D^{\prime}(\omega) \subset D(\omega)$ for all $\omega \in \Omega$, then $D^{\prime} \in \mathcal{D}$.

Definition 2.3. A family $\mathcal{A}=\{A(\omega), \omega \in \Omega\}$ of nonempty measurable compact subsets $\mathcal{A}(\omega)$ of $\mathbb{E}$ is called $\varphi$ - invariant if $\varphi(t, \omega, \mathcal{A}(\omega))=\mathcal{A}\left(\theta_{t} \omega\right)$ for all $t \in \mathbb{R}^{+}$and is called a random attractor if in addition it is pathwise pullback attracting in the sense that

$$
H_{d}^{*}\left(\varphi\left(t, \theta_{-t} \omega, D\left(\theta_{-t} \omega\right)\right), \mathcal{A}(\omega)\right) \rightarrow 0 \quad \text { as } t \rightarrow \infty
$$

for all $D \in \mathcal{D}$. Here $H_{d}^{*}$ is the Hausdorff semi-distance on $\mathbb{E}$.

Definition 2.4. A random variable $u: \Omega \mapsto \mathbb{E}$ is said to be a random equilibrium of the $R D S$ $\varphi$ if it is invariant under $\varphi$, i.e. if

$$
\varphi(t, \omega) u(\omega)=u\left(\theta_{t} \omega\right) \quad \text { for all } \quad t \geq 0 \quad \text { and all } \quad \omega \in \Omega
$$

Definition 2.5. A random variable $r: \Omega \rightarrow \mathbb{R}$ is called tempered if

$$
\lim _{t \rightarrow \pm \infty} \frac{\log \left|r\left(\theta_{t} \omega\right)\right|}{|t|}=0 \quad \mathbb{P}-\text { a.s. }
$$

and a random set $\{D(\omega), \omega \in \Omega\}$ with $D(\omega) \subset \mathbb{E}$ is called tempered if it is contained in the ball $\{x \in \mathbb{R}:|x| \leq r(\omega)\}$, where $r$ is a tempered random variable.

Here we will always work with the attracting universe given by the tempered random sets.

Definition 2.6. A family $\hat{B}=\{B(\omega), \omega \in \Omega\}$ is said to be pullback absorbing if for every $D(\omega) \in \mathcal{D}$, there exists $T_{D}(\omega) \geq 0$ such that

$$
\varphi\left(t, \theta_{-t} \omega, D\left(\theta_{-t} \omega\right)\right) \subset B(\omega) \quad \forall t \geq T_{D}(\omega)
$$

The following result (cf. Proposition 9.3.2 in [31], Theorem 2.2 in [33]) guarantees the existence of a random attractor.

Theorem 2.7. Let $(\theta, \varphi)$ be a continuous $R D S$ on $\Omega \times \mathbb{E}$. If there exists a pullback absorbing family $\hat{B}=\{B(\omega), \omega \in \Omega\}$ such that, for every $\omega \in \Omega, B(\omega)$ is compact and $B(\omega) \in \mathcal{D}$, then the $\operatorname{RDS}(\theta, \varphi)$ has a random attractor 


$$
\mathcal{A}(\omega)=\bigcap_{\tau>0} \overline{\bigcup_{t \geqslant \tau} \varphi\left(t, \theta_{-t} \omega\right) B\left(\theta_{-t} \omega\right)}
$$

Note that if the random attractor consists of singleton sets, i.e. $\mathcal{A}(\omega)=\left\{u^{*}(\omega)\right\}$ for some random variable $u^{*}$, then $u^{*}(t)(\omega)=u^{*}(t)\left(\theta_{t} \omega\right)$ is a stationary stochastic process.

\section{FitzHugh-Nagumo Lattice Equations with Fractional Brown- ian Motions}

We now recall the definition of a fractional Brownian motion. Given $H \in(0,1)$, a continuous centered Gaussian process $\beta^{H}(t), t \in \mathbb{R}$, with the covariance function

$$
\mathbf{E} \beta^{H}(t) \beta^{H}(s)=\frac{1}{2}\left(|t|^{2 H}+|s|^{2 H}-|t-s|^{2 H}\right), \quad t, s \in \mathbb{R}
$$

is called a two-sided one-dimensional $\mathrm{fBm}$, and $H$ is the Hurst parameter. For $H=1 / 2, \beta$ is a standard Brownian motion, while for $H \neq 1 / 2$, it is neither a semimartingale nor a Markov process. Moreover,

$$
\mathbf{E}\left|\beta^{H}(t)-\beta^{H}(s)\right|^{2}=|t-s|^{2 H}, \text { for all } s, t \in \mathbb{R} .
$$

Here, we assume that $H \in(1 / 2,1)$ throughout the paper. When $H \in(0,1 / 2)$ we cannot define the stochastic integral by a generalized Stieljes integral and, therefore, dealing with such values of the Hurst parameter seems to be much more complicated. It is worth mentioning that when $H=1 / 2$ the $\mathrm{fBm}$ becomes the standard Wiener process, the random dynamical system generated by the (similar) stochastic FitzHugh-Nagumo lattice equations has been studied in [10, 24].

Using the definition of $\beta^{H}(t)$, Kolmogorov's theorem ensures that $\beta^{H}$ has a continuous version, and almost all the paths are Hölder continuous of any order $H^{\prime} \in(0, H)$ (see [34]). Thus, let $\mathbb{E}=\ell^{2} \times \ell^{2}$ and norm $\|\cdot\|_{\mathbb{E}}$, we can consider the canonical interpretation of an $\mathrm{fBm}$ : denote $\Omega=C_{0}\left(\mathbb{R}, \ell^{2}\right)$, the space of continuous functions on $\mathbb{R}$ with values in $\ell^{2}$ such that $\omega(0)=0$, equipped with the compact open topology. Let $\mathcal{F}$ be the associated Borel- $\sigma$-algebra and $\mathbb{P}$ the distribution of the $\mathrm{fBm} \beta^{H}$, and $\left\{\theta_{t}\right\}_{t \in \mathbb{R}}$ be the flow of Wiener shifts such that

$$
\theta_{t} \omega(\cdot)=\omega(\cdot+t)-\omega(t), \quad t \in \mathbb{R}
$$

Due to [17]-35], we know that the quadruple $(\Omega, \mathcal{F}, \mathbb{P}, \theta)$ is an ergodic metric dynamical system. Furthermore, it holds that

$$
\begin{gathered}
\beta^{H}(\cdot, \omega)=\omega(\cdot), \\
\beta^{H}\left(\cdot, \theta_{s} \omega\right)=\beta^{H}(\cdot+s, \omega)-\beta^{H}(s, \omega) \\
=\omega(\cdot+s)-\omega(s) .
\end{gathered}
$$


For $u=\left(u_{i}\right)_{i \in \mathbb{Z}} \in \ell^{2}$, define $\mathbb{A}, \mathbb{B}, \mathbb{B}^{*}$ to be linear operators from $\ell^{2}$ to $\ell^{2}$ as follows:

$$
\begin{aligned}
& (\mathbb{A} u)_{i}=-u_{i-1}+2 u_{i}-u_{i+1}, \\
& (\mathbb{B} u)_{i}=u_{i+1}-u_{i}, \quad\left(\mathbb{B}^{*} u\right)_{i}=u_{i-1}-u_{i}, \quad i \in \mathbb{Z} .
\end{aligned}
$$

It is easy to show that $\mathbb{A}=\mathbb{B B}^{*}=\mathbb{B}^{*} \mathbb{B},\left(\mathbb{B}^{*} u, u^{\prime}\right)=\left(u, \mathbb{B} u^{\prime}\right)$ for all $u, u^{\prime} \in \ell^{2}$, which implies that $(\mathbb{A} u, u) \geq 0$.

Let $W_{1}(t) \equiv W_{1}(t, \omega)=\sum_{i \in \mathbb{Z}} a_{i} \omega_{i}(t) e^{i}$ and $W_{2}(t) \equiv W_{2}(t, \omega)=\sum_{i \in \mathbb{Z}} b_{i} \omega_{i}(t) e^{i}$, here $\left(e^{i}\right)_{i \in \mathbb{Z}} \in$ $\ell^{2}$ denote the element having 1 at position $i$ and the other components 0. Then SLDS (1.1) with initial conditions can be rewritten as pathwise Riemann-Stieltjes integral equations in $\mathbb{E}$

$$
\left\{\begin{array}{l}
u(t)=u(0)+\int_{0}^{t}(-\mathbb{A} u(s)-\lambda u(s)+f(u(s))-v(s)) d s+W_{1}(t), \\
v(t)=v(0)+\int_{0}^{t}(\varrho u(s)-\sigma v(s)) d s+W_{2}(t), \\
u(0)=u_{0}=\left(u_{i 0}\right)_{i \in \mathbb{Z}}, \quad v(0)=v_{0}=\left(v_{i 0}\right)_{i \in \mathbb{Z}},
\end{array}\right.
$$

where $u=\left(u_{i}\right)_{i \in \mathbb{Z}}, \lambda, \varrho$ and $\sigma$ are positive constants, $a=\left(a_{i}\right)_{i \in \mathbb{Z}} \in \ell^{2}, b=\left(b_{i}\right)_{i \in \mathbb{Z}} \in \ell^{2}$ and $\left\{\omega_{i}=\beta_{i}^{H}: i \in \mathbb{Z}\right\}$ are independent two-sided fractional Brownian motions with Hurst parameter $H \in(1 / 2,1), f(u)=\left(f_{i}\left(u_{i}\right)\right)_{i \in \mathbb{Z}}$ is a nonlinear smooth function satisfies a one-sided dissipative Lipschitz condition

$$
(f(u)-f(v), u-v) \leq-\gamma\|u-v\|^{2} \text { for all } u, v \in \mathbb{R}
$$

and the polynomial growth condition

$$
|f(u)| \leq c_{f}\left(|u|^{2 p+1}+1\right) \text { for all } u \in \mathbb{R}
$$

where $\gamma$ is a positive constant, $p$ is a positive integer.

In addition we could consider a more general dissipativity condition, which would lead to nontrivial setvalued random attractors, we will restrict here to the dissipativity condition (3.3). When system (1.1) with Hurst parameter $H=1 / 2$ and under conditions (3.3) and (3.4), we can apply the result of Theorem 3.1 in [14], i.e. the combination of the existence of a bounded closed random absorbing set and the property of random asymptotic nullity to get the existence of a compact random attractor. Moreover, we have the following results:

Lemma 3.1. There exists positive random constants $\left(\tilde{\rho}_{i}(\omega)\right)_{i \in \mathbb{Z}} \in \ell^{2}$ and $\rho(\omega)=\|\tilde{\rho}(\omega)\|$ such

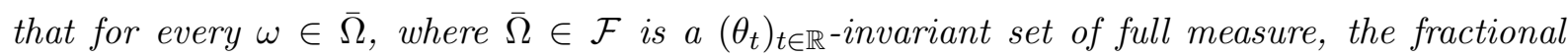
Brownian motions are well defined for $t \in \mathbb{R}$ in $\ell^{2}$ satisfying

$$
\left\|W_{j}(t)\right\|^{2} \leq 2 \max \left\{\|a\|^{2},\|b\|^{2}\right\} \rho^{2}(\omega)\left(1+|t|^{4}\right), \quad j=1,2 .
$$


Proof. Obviously.

Proposition 3.2. Let the above assumptions on $f$ be satisfied and $T>0$. Then system (3.2) has a unique pathwise solution $\Psi=(\Psi(t))_{t \geq 0}=(u(t), v(t))_{t \geq 0}$. Furthermore, the solution satisfies

$$
\begin{aligned}
\sup _{t \in[0, T]}\|\Psi(t)\|_{\mathbb{E}}^{2} & \leq M\left[\left\|\Psi_{0}\right\|_{\mathbb{E}}^{2}+\sup _{t \in[0, T]}\left(\left\|W_{1}(t)\right\|^{2}+\left\|W_{2}(t)\right\|^{2}\right)\right. \\
& \left.+\int_{0}^{T}\left(\left\|W_{1}(s)\right\|^{4 p+2}+\left\|W_{1}(s)\right\|^{2}+\left\|W_{2}(s)\right\|^{2}+1\right) d s\right],
\end{aligned}
$$

where $M$ is a positive constant independent of $T$.

Proof. Let $\tilde{u}(t)=u(t)-W_{1}(t)$ and $\tilde{v}(t)=v(t)-W_{2}(t)$, system (3.2) has a solution $\Psi=(\Psi(t))_{t \geq 0}$ for all $\omega \in \Omega$ if and only if the following system

$$
\left\{\begin{array}{c}
\tilde{u}(t)=u(0)+\int_{0}^{t}\left(-\mathbb{A} \tilde{u}(s)-\lambda \tilde{u}(s)+f\left(\tilde{u}(s)+W_{1}(s)\right)\right. \\
\left.-\tilde{v}(s)-\mathbb{A} W_{1}(s)-\lambda W_{1}(s)-W_{2}(s)\right) d s \\
\tilde{v}(t)=v(0)+\int_{0}^{t}\left(\varrho \tilde{u}(s)-\sigma \tilde{v}(s)-\varrho W_{1}(s)-\sigma W_{2}(s)\right) d s \\
u(0)=u_{0}=\left(\tilde{u}_{i 0}\right)_{i \in \mathbb{Z}}, \quad v(0)=v_{0}=\left(\tilde{v}_{i 0}\right)_{i \in \mathbb{Z}}
\end{array}\right.
$$

has a unique pathwise solution for $t \in[0, T]$. However, since the integrand is pathwise continuous, the fundamental theorem of calculus says that the left hand side of (3.5) is pathwise differentiable. Thus, for a fixed $\omega \in \Omega$, system (3.5) is the pathwise system of random ODEs

$$
\left\{\begin{array}{c}
\frac{d \tilde{u}(t)}{d t}=-\mathbb{A} \tilde{u}(t)-\lambda \tilde{u}(t)+f\left(\tilde{u}(t)+W_{1}(t)\right) \\
\quad-\tilde{v}(t)-\mathbb{A} W_{1}(t)-\lambda W_{1}(t)-W_{2}(t) \\
\frac{d \tilde{v}(t)}{d t}=\varrho \tilde{u}(t)-\sigma \tilde{v}(t)+\varrho W_{1}(t)-\sigma W_{2}(t) \\
u(0)=u_{0}=\left(\tilde{u}_{i 0}\right)_{i \in \mathbb{Z}}, \quad v(0)=v_{0}=\left(\tilde{v}_{i 0}\right)_{i \in \mathbb{Z}}
\end{array}\right.
$$

Since $f(u)$ is a continuous function, and the assumptions on $f$ are satisfied, by the standard argument on existence theorem for ODEs, it follows that system (3.6) possesses a local solution in a small interval $[0, \tau(\omega)]$, which means system (3.2) has a unique local solution in the same small interval $[0, \tau(\omega)]$. Here, we remain to show that the local solution is a global one.

For a fixed $\omega \in \Omega$, by taking the inner product of (3.6) with $(\tilde{u}, \tilde{v})$ in $\mathbb{E}$, it follows that

$$
\begin{gathered}
\|\tilde{u}(t)\|^{2}+\frac{1}{\varrho}\|\tilde{v}(t)\|^{2}=\left\|\tilde{u}_{0}\right\|^{2}+\frac{1}{\varrho}\left\|\tilde{v}_{0}\right\|^{2}+2 \int_{0}^{t}(-\mathbb{A} \tilde{u}(s), \tilde{u}(s)) d s \\
+2 \int_{0}^{t}\left(f\left(\tilde{u}(s)+W_{1}(s)\right), \tilde{u}(s)\right) d s+2 \int_{0}^{t}\left(-\mathbb{A} W_{1}(s), \tilde{u}(s)\right) d s \\
-2 \int_{0}^{t}\left(\lambda W_{1}(s), \tilde{u}(s)\right) d s-2 \int_{0}^{t}\left(W_{2}(s), \tilde{u}(s)\right) d s \\
-2 \lambda \int_{0}^{t}\|\tilde{u}(s)\|^{2} d s-\frac{2 \sigma}{\varrho} \int_{0}^{t}\|\tilde{v}(s)\|^{2} d s \\
-\frac{2 \sigma}{\varrho} \int_{0}^{t}\left(W_{2}(s), \tilde{v}(s)\right) d s+2 \int_{0}^{t}\left(W_{1}(s), \tilde{v}(s)\right) d s .
\end{gathered}
$$


By (3.3) and (3.4), we obtain that

$$
\begin{aligned}
& 2\left(f\left(\tilde{u}(s)+W_{1}(s)\right), \tilde{u}(s)\right) \\
= & 2\left(f\left(\tilde{u}(s)+W_{1}(s)\right), \tilde{u}(s)+W_{1}(s)\right)-2\left(f\left(\tilde{u}(s)+W_{1}(s), W_{1}(s)\right)\right. \\
\leq & -\gamma\left\|\tilde{u}(s)+W_{1}(s)\right\|^{2}+2 \mid f\left(\tilde{u}(s)+W_{1}(s) \| W_{1}(s) \mid\right. \\
\leq & c_{1}\left(\left\|W_{1}(s)\right\|^{4 p+2}+\left\|W_{1}(s)\right\|^{2}+1\right),
\end{aligned}
$$

where $c_{1}$ is a positive constant depends on $\gamma, c_{f}$ and $p$. By Young's inequality, it yields that

$$
\begin{aligned}
& 2(-\mathbb{A} \tilde{u}(s), \tilde{u}(s))+2\left(f\left(\tilde{u}(s)+W_{1}(s)\right), \tilde{u}(s)\right)+2\left(-\mathbb{A} W_{1}(s), \tilde{u}(s)\right) \\
\leq & \lambda\|\tilde{u}(s)\|^{2}+c_{2}\left(\left\|W_{1}(s)\right\|^{2}+\left\|W_{2}(s)\right\|^{2}\right),
\end{aligned}
$$

where $c_{2}$ is a positive constant depends on $\lambda$, and

$$
\begin{aligned}
& \frac{2 \sigma}{\varrho}\left(W_{2}(s), \tilde{v}(s)\right)+2\left(W_{1}(s), \tilde{v}(s)\right) \\
\leq & \frac{\sigma}{\varrho}\|\tilde{v}(s)\|^{2}+c_{3}\left(\left\|W_{1}(s)\right\|^{2}+\left\|W_{2}(s)\right\|^{2}\right),
\end{aligned}
$$

where $c_{3}$ is a positive constant depends on $\varrho$ and $\sigma$. Let $\alpha=\min \{\lambda, \sigma\}$ and combine (3.8)(3.10) with (3.7), for $t \geq 0$, we get

$$
\begin{aligned}
\|\tilde{u}(t)\|^{2}+ & \frac{1}{\varrho}\|\tilde{v}(t)\|^{2} \leq\left\|\tilde{u}_{0}\right\|^{2}+\frac{1}{\varrho}\left\|\tilde{v}_{0}\right\|^{2}-\alpha \int_{0}^{t}\left(\|\tilde{u}(s)\|^{2}+\frac{1}{\varrho}\|\tilde{v}(s)\|^{2}\right) d s \\
& +c \int_{0}^{t}\left(\left\|W_{1}(s)\right\|^{4 p+2}+\left\|W_{1}(s)\right\|^{2}+\left\|W_{2}(s)\right\|^{2}+1\right) d s \\
\leq & \left\|\tilde{u}_{0}\right\|^{2}+\frac{1}{\varrho}\left\|\tilde{v}_{0}\right\|^{2} \\
& +c \int_{0}^{t}\left(\left\|W_{1}(s)\right\|^{4 p+2}+\left\|W_{1}(s)\right\|^{2}+\left\|W_{2}(s)\right\|^{2}+1\right) d s,
\end{aligned}
$$

where $c$ is a positive constant depends on $\varrho, \sigma, \lambda, \gamma, c_{f}$ and $p$. Hence, from (3.11), we know that $\|\tilde{u}(t)\|^{2}+\frac{1}{\varrho}\|\tilde{v}(t)\|^{2}$ is bounded by a continuous function, which implies the global existence of a solution on interval $[0, T]$. Furthermore, for all $\omega \in \Omega$, it follows that

$$
\begin{gathered}
\sup _{t \in[0, T]}\left(\|u(t)\|^{2}+\frac{1}{\varrho}\|v(t)\|^{2}\right)=\sup _{t \in[0, T]}\left(\left\|\tilde{u}(t)+W_{1}(t)\right\|^{2}+\frac{1}{\varrho}\left\|\tilde{v}(t)+W_{2}(t)\right\|^{2}\right) \\
\leq 2\left(\left\|u_{0}\right\|^{2}+\frac{1}{\varrho}\left\|v_{0}\right\|^{2}\right)+2 \sup _{t \in[0, T]}\left(\left\|W_{1}(t)\right\|^{2}+\frac{1}{\varrho}\left\|W_{2}(t)\right\|^{2}\right) \\
+2 c \int_{0}^{T}\left(\left\|W_{1}(s)\right\|^{4 p+2}+\left\|W_{1}(s)\right\|^{2}+\left\|W_{2}(s)\right\|^{2}+1\right) d s .
\end{gathered}
$$

According to Lemma 3.1 , we know that the right side of $(\underline{3.12})$ is well defined. Let $\tilde{\alpha}=\frac{\max \left\{1, \frac{1}{\varrho}\right\}}{\min \left\{1, \frac{1}{\varrho}\right\}}$, the proof is complete. 
Proposition 3.3. The solution of (3.2) determinants a continuous random dynamical system $\varphi: \mathbb{R}^{+} \times \Omega \times \mathbb{E} \rightarrow \mathbb{E}$, which is given by

$$
\varphi\left(t, \omega, \Psi_{0}\right)=\Psi_{0}+\int_{0}^{t} G(\Psi(s)) d s+\eta(t, \omega) \text { for } t \geq 0
$$

where $G(\Psi(t))=L \Psi(t)+F(\Psi(t))$ and

$$
L=\left(\begin{array}{cc}
-\mathbb{A}-\lambda & -1 \\
\varrho & -\sigma
\end{array}\right), F(\Psi)=\left(\begin{array}{c}
f(u) \\
0
\end{array}\right), \eta(t, \omega)=\left(\begin{array}{c}
W_{1}(t, \omega) \\
W_{2}(t, \omega)
\end{array}\right) .
$$

Proof. For the need of making the relations clear between $G(\Psi(t))(\cdot)$ and $\omega$, we write $G(\Psi(t))(\omega)$ instead if necessary. Note that (3.1) is satisfied for $\omega \in \Omega$ and by the definition of $\left(\theta_{t}\right)_{t \in \mathbb{R}}$, we have the property

$$
\eta(\tau+t, \omega)=\eta\left(\tau, \theta_{t} \omega\right)+\eta(t, \omega) \text { for all } t, \tau \in \mathbb{R} .
$$

By Proposition 3.2 we know that $\varphi$ solves (3.2), thus $\varphi$ is measurable and satisfies $\varphi(0, \omega, \cdot)=\mathrm{id}_{\mathbb{E}}$. It remains to verify that the cocycle property in Definition 2.2 . Let $t, \tau \in \mathbb{R}^{+}, \omega \in \Omega$ and $\Psi_{0} \in \mathbb{E}$, it yields from (3.1) that

$$
\begin{aligned}
& \varphi\left(t+\tau, \omega, \Psi_{0}\right) \\
= & \Psi_{0}+\int_{0}^{t+\tau} G(\Psi(s))(\omega) d s+\eta(t+\tau, \omega) \\
= & \Psi_{0}+\int_{0}^{t} G(\Psi(s))(\omega) d s+\eta(t, \omega)+\int_{t}^{t+\tau} G(\Psi(s))(\omega) d s+\eta\left(\tau, \theta_{t} \omega\right) \\
= & \Psi(t)+\int_{0}^{\tau} G(\Psi(s))\left(\theta_{t} \omega\right) d s+\eta\left(\tau, \theta_{t} \omega\right) \\
= & \varphi\left(\tau, \theta_{t} \omega, \cdot\right) \circ \varphi\left(t, \omega, \Psi_{0}\right),
\end{aligned}
$$

which completes the proof.

\section{Existence of a Random Attractor}

In this section, we will prove the existence of a random attractor for the RDS defined in Proposition 3.3 . Sometimes, for the need of making the relations between $\bar{u}(\cdot)$ (or $\bar{v}, \Psi, \bar{\Phi}$ ) and $\omega$ more explicitly, we will write $\bar{u}(\omega)$ (or $\bar{v}(\omega), \Psi(\omega), \bar{\Phi}(\omega)$ ) instead if necessary.

Consider the following fractional Ornstein-Uhlenbeck processes

$$
d u(t)=-\lambda u(t) d t+d W_{1}(t), d v(t)=-\sigma v(t) d t+d W_{2}(t),
$$

where $\lambda, \sigma$ defined in (3.2) and $W_{1}(t), W_{2}(t)$ denote one-dimensional fractional Brownian motions. They have the explicit solutions

$$
u(t)=u_{0} e^{-\lambda t}+e^{-\lambda t} \int_{0}^{t} e^{\lambda s} d W(s), v(t)=v_{0} e^{-\sigma t}+e^{-\sigma t} \int_{0}^{t} e^{\sigma s} d W(s) .
$$


Take the pathwise pullback limits, we get the stochastic stationary solutions

$$
\bar{u}(t)=e^{-\lambda t} \int_{-\infty}^{t} e^{\lambda s} d W(s), \bar{v}(t)=e^{-\sigma t} \int_{-\infty}^{t} e^{\sigma s} d W(s), \quad t \in \mathbb{R},
$$

which are called the fractional Ornstein-Uhlenbeck solutions. We have the following properties:

Lemma 4.1. There exists positive random constants $\left(\check{\rho}_{i}(\omega)\right)_{i \in \mathbb{Z}},\left(\hat{\rho}_{i}(\omega)\right)_{i \in \mathbb{Z}} \in \ell^{2}$ and $\check{\rho}^{2}(\omega)=$ $16 \sum_{i \in \mathbb{Z}} a_{i}^{2} \check{\rho}_{i}^{2}(\omega), \hat{\rho}^{2}(\omega)=16 \sum_{i \in \mathbb{Z}} b_{i}^{2} \hat{\rho}_{i}^{2}(\omega)$ for all $\omega \in \Omega$, the Riemann-Stieltjes integrals in (4.3) are well defined in $\ell^{2}$. Moreover, for all $\omega \in \Omega, t \in \mathbb{R}$, we have

$$
\left\|e^{-\lambda t} \int_{-\infty}^{t} e^{\lambda s} d W_{1}(s)\right\| \leq \check{\rho}(\omega)(1+|t|)^{2},\left\|e^{-\sigma t} \int_{-\infty}^{t} e^{\sigma s} d W_{2}(s)\right\| \leq \hat{\rho}(\omega)(1+|t|)^{2} .
$$

Proof. By the Lemma 1 in [26], we can easily get the conclusion.

Now, we are in the position to state the main result.

Theorem 4.2. Assume that the conditions on $f$ are satisfied. Then the random dynamical system $\varphi$ has a unique random equilibrium, which constitutes a singleton sets random attractor.

Proof. Let $\Psi(t)=(u(t), v(t)), \Phi(t)=(\tilde{u}(t), \tilde{v}(t))$ be any two solutions of system (1.1). Their sample paths are not differentiable, but the difference satisfies pathwise for $t \geq 0$,

$$
\Psi(t)-\Phi(t)=\Psi_{0}-\Phi_{0}+\int_{0}^{t}(L(\Psi(s)-\Phi(s))+(F(\Psi(s))-F(\Phi(s))) d s,
$$

and again, since the integrand is pathwise continuous, the fundamental theorem of calculus indicates that the left hand side is pathwise differentiable and satisfies

$$
\frac{d}{d t}(\Psi(t)-\Phi(t))=L(\Psi(t)-\Phi(t))+F(\Psi(t))-F(\Phi(t)), t \geq 0 .
$$

Recall that $\alpha=\min \{\lambda, \sigma\}$, we obtain from (4.4) that

$$
\begin{aligned}
\frac{d}{d t}\|\Psi(t)-\Phi(t)\|_{\mathbb{E}}^{2}= & 2(\Psi(t)-\Phi(t), L(\Psi(t)-\Phi(t)))_{\mathbb{E}} \\
& +2(\Psi(t)-\Phi(t), F(\Psi(t))-F(\Phi(t)))_{\mathbb{E}} \\
\leq & -2 \alpha\|\Psi(t)-\Phi(t)\|_{\mathbb{E}}^{2} .
\end{aligned}
$$

Thus pathwise we have

$$
\|\Psi(t)-\Phi(t)\|_{\mathbb{E}}^{2} \leq\left\|\Psi_{0}-\Phi_{0}\right\|_{\mathbb{E}}^{2} e^{-2 \alpha t} \rightarrow 0, \text { as } t \rightarrow \infty
$$

That is to say that all solutions converge pathwise forward to each other in time. 
Now, we want to know where the solution will converge to. Let $\bar{\Phi}(t)=(\bar{u}(t), \bar{v}(t))$. We consider the difference $\Psi(t)-\bar{\Phi}(t)$. Since their paths are continuous, the difference is pathwise differentiable and satisfies the integral equation for $t \geq 0$,

$$
\Psi(t)-\bar{\Phi}(t)=\Psi_{0}-\bar{\Phi}_{0}+\int_{0}^{t}(L(\Psi(s)-\bar{\Phi}(s))+(F(\Psi(s))-F(\bar{\Phi}(s))) d s,
$$

which is equivalent to the pathwise differential equation

$$
\frac{d}{d t}(\Psi(t)-\bar{\Phi}(t))=L(\Psi(s)-\bar{\Phi}(s))+(F(\Psi(s))-F(\bar{\Phi}(s)), \quad t \geq 0 .
$$

That is to consider the following system

$$
\left\{\begin{array}{l}
\frac{d}{d t}(u(t)-\bar{u}(t))=-\mathbb{A} u(t)-\lambda(u(t)-\bar{u}(t))+f(u(t))-v(t) \\
\frac{d}{d t}(v(t)-\bar{v}(t))=\varrho u(t)-\sigma(v(t)-\bar{v}(t))
\end{array}\right.
$$

By taking the inner product in $\mathbb{E}$, we get

$$
\begin{gathered}
\frac{d}{d t}\left(\|u(t)-\bar{u}(t)\|^{2}+\frac{1}{\varrho}\|v(t)-\bar{v}(t)\|^{2}\right) \\
=2(-\mathbb{A} u(t), u(t)-\bar{u}(t))+2(f(u), u(t)-\bar{u}(t)) \\
-2(v(t), u(t)-\bar{u}(t))+2(u(t), v(t)-\bar{v}(t)) \\
-2 \lambda\|u(t)-\bar{u}(t)\|^{2}-\frac{2 \sigma}{\varrho}\|v(t)-\bar{v}(t)\|^{2} .
\end{gathered}
$$

We know that

$$
\begin{gathered}
2(-\mathbb{A} u(t), u(t)-\bar{u}(t))=2(-\mathbb{A}(u(t)-\bar{u}(t)), u(t)-\bar{u}(t)) \\
+2(\mathbb{A} \bar{u}(t), u(t)-\bar{u}(t)) \\
\leq \frac{\lambda}{2}\|u(t)-\bar{u}(t)\|^{2}+\frac{32}{\lambda}\|\bar{u}(t)\|^{2} \\
2(f(u), u(t)-\bar{u}(t))=2(f(u)-f(\bar{u}), u(t)-\bar{u}(t)) \\
+2(f(\bar{u}), u(t)-\bar{u}(t)) \\
\leq-\gamma\|u(t)-\bar{u}(t)\|^{2}+\frac{\lambda}{2}\|u(t)-\bar{u}(t)\|^{2}+\frac{8}{\lambda}\|f(\bar{u})\|^{2}, \\
-2(v(t), u(t)-\bar{u}(t))+2(u(t), v(t)-\bar{v}(t)) \\
\leq \gamma\|u(t)-\bar{u}(t)\|^{2}+\frac{4}{\gamma}\|\bar{v}(t)\|^{2}+\frac{\sigma}{\varrho}\|v(t)-\bar{v}(t)\|^{2}+\frac{4 \varrho}{\sigma}\|\bar{u}(t)\|^{2} .
\end{gathered}
$$

Combine the three inequalities above with (4.6), we have

$$
\begin{gathered}
\frac{d}{d t}\left(\|u(t)-\bar{u}(t)\|^{2}+\frac{1}{\varrho}\|v(t)-\bar{v}(t)\|^{2}\right) \\
\leq \quad-\lambda\|u(t)-\bar{u}(t)\|^{2}-\frac{\sigma}{\varrho}\|v(t)-\bar{v}(t)\|^{2} \\
+c_{4}\left(\|\bar{u}(t)\|^{2}+\|\bar{v}(t)\|^{2}+\|f(\bar{u})\|^{2}\right),
\end{gathered}
$$


where $c_{4}$ is a positive constant depends on $\lambda, \varrho$ and $\sigma$. Then we obtain

$$
\frac{d}{d t}\|\Psi(t)-\bar{\Phi}(t)\|_{\mathbb{E}}^{2} \leq-\alpha\|\Psi(t)-\bar{\Phi}(t)\|_{\mathbb{E}}^{2}+c_{4}\left(\|\bar{u}(t)\|^{2}+\|\bar{v}(t)\|^{2}+\|f(\bar{u})\|^{2}\right),
$$

and hence

$$
\begin{aligned}
\|\Psi(t)-\bar{\Phi}(t)\|_{\mathbb{E}}^{2} \leq\left\|\Psi_{0}(\omega)-\bar{\Phi}_{0}(\omega)\right\|^{2} e^{-\alpha t} & \\
& +c_{4} e^{-\alpha t} \int_{0}^{t} e^{\alpha s}\left(\|\bar{u}(s)\|^{2}+\|\bar{v}(s)\|^{2}+\|f(\bar{u}(s))\|^{2}\right) d s
\end{aligned}
$$

Let us check that the family of balls centered on $\bar{\Phi}_{0}(\omega)$ with the random radius

$$
R(\omega):=\sqrt{1+c_{4} \int_{-\infty}^{0} e^{\alpha s}\left(\|\bar{u}(s)(\omega)\|^{2}+\|\bar{v}(s)(\omega)\|^{2}+\|f(\bar{u}(s)(\omega))\|^{2}\right) d s}
$$

is a pullback absorbing family for the random dynamical system generated by system (1.1).

Due to the assumptions on $f$ and Lemma 4.1, the radius defined in (4.9) is well defined. Now, by replacing $\omega$ by $\theta_{-t} \omega$ in (4.8), we get

$$
\begin{aligned}
& \left\|\Psi\left(\theta_{-t} \omega\right)-\bar{\Phi}\left(\theta_{-t} \omega\right)\right\|_{\mathbb{E}}^{2} \\
\leq & \left\|\Psi_{0}\left(\theta_{-t} \omega\right)-\bar{\Phi}_{0}\left(\theta_{-t} \omega\right)\right\|_{\mathbb{E}}^{2} e^{-\alpha t} \\
& +c_{4} \int_{0}^{t} e^{\alpha(s-t)}\left(\left\|\bar{u}(s)\left(\theta_{-t} \omega\right)\right\|^{2}+\left\|\bar{v}(s)\left(\theta_{-t} \omega\right)\right\|^{2}+\left\|f\left(\bar{u}(s)\left(\theta_{-t} \omega\right)\right)\right\|^{2}\right) d s \\
= & \left\|\Psi_{0}\left(\theta_{-t} \omega\right)-\bar{\Phi}_{0}\left(\theta_{-t} \omega\right)\right\|_{\mathbb{E}}^{2} e^{-\alpha t} \\
& +c_{4} \int_{-t}^{0} e^{\alpha s}\left(\|\bar{u}(s)(\omega)\|^{2}+\|\bar{v}(s)(\omega)\|^{2}+\|f(\bar{u}(s)(\omega))\|^{2}\right) d s .
\end{aligned}
$$

The last term in (4.10) due to $\bar{u}(s)\left(\theta_{-t} \omega\right)=\bar{u}_{0}\left(\theta_{s-t} \omega\right)=\bar{u}(s-t)(\omega)$ and $\bar{v}(s)\left(\theta_{-t} \omega\right)=$ $\bar{v}_{0}\left(\theta_{s-t} \omega\right)=\bar{v}(s-t)(\omega)$ which deduced from that $(\bar{u}(t))_{t \in \mathbb{R}}$ and $(\bar{v}(t))_{t \in \mathbb{R}}$ are stationary processes. The conclusion now follows as $t \rightarrow \infty$.

Because of the stationarity and Lemma 4.1, we have $e^{-\alpha t}\left\|\bar{\Phi}_{0}\left(\theta_{-t} \omega\right)\right\|_{\mathbb{E}}^{2}=e^{-\alpha t}\|\bar{\Phi}(-t)(\omega)\|_{\mathbb{E}}^{2} \rightarrow$ 0 as $t \rightarrow \infty$. Then we have the pullback absorption

$$
\left\|\Psi\left(\theta_{-t} \omega\right)\right\|_{\mathbb{E}}^{2} \leq\left\|\bar{\Phi}_{0}(\omega)\right\|_{\mathbb{E}}^{2}+R^{2}(\omega), \quad \forall t \geq T_{\mathcal{D}(\omega)}
$$

So, we have the stationary random process $\tilde{\Phi}(t)(\omega):=\tilde{\Phi}_{0}\left(\theta_{t} \omega\right)$, which pathwise attracts all other solutions in both forward and pullback senses, is a random equilibrium. Now, we define a singleton sets $\mathcal{A}=\{A(\omega), \omega \in \Omega\}=\left\{\tilde{\Phi}_{0}(\omega)\right\}$, i.e. the singleton sets is formed by the random equilibrium. Here, we remain to show that the singleton sets turns out to be a random attractor. According to Definition 2.3 , we can easily get the compactness, invariance and attraction (implied by absorbtion). The proof is complete. 


\section{Conclusions}

We studied the stochastic FitzHugh-Nagumo equations driven by fractional Brownian motion. The existence of the random attractor formed by the unique random equilibrium turns out to be a single sets random attractor, which differs from the results obtained in [10] and [24] where the same system is driven by white noises. The methodology can be used to deal with other stochastic lattice systems, which is a topic that will be the focus of further research.

\section{Acknowledgements}

The authors would like to express their sincere thanks to the anonymous referees for their time and helpful comments and suggestions, which have largely improved the presentation of this paper.

\section{References}

[1] Bates P W, Lu K, Wang B. Attractors for lattice dynamical systems, Int. J. Bifurcation Chaos 11 (2001) 143-153.

[2] Wang B. Dynamics of systems on infinite lattices, J. Differ. Eqns. 221 (2006) 224-245.

[3] Zhou S. Attractors for second order lattice dynamical systems, J. Differ. Eqns. 179 (2002) 605-624.

[4] Zhou S. Attractors and approximations for lattice dynamical systems, J. Differ. Eqns. 200 (2004) 342-368.

[5] Zhou S, Shi W. Attractors and dimension of dissipative lattice systems, J. Differ. Eqns. 224 (2006) 172-204.

[6] Bates P W, Lisei H, Lu K. Attractors for stochastic lattice dynamical systems, Stoch. Dyn. 6 (2006) $1-21$.

[7] Caraballo T, Lu K. Attractors for stochastic lattice dynamical systems with a multiplicative noise, Front. Math. China 3(3) (2008) 317-335.

[8] Lv Y, Sun J. Dynamical behavior for stochastic lattice systems, Chaos Solitons Fractals 27 (2006) 1080-1090. 
[9] Lv Y, Sun J. Asymptotic behavior of stochastic discrete complex Ginzburg-Landau equations, Physica D 221 (2006) 157-169.

[10] Huang J. The random attractor of stochastic FitzHugh-Nagumo equations in an infinite lattice with white noises, Physica D 233 (2007) 83-94.

[11] Wang X, Li S, Xu D. Random attractors for second-order stochastic lattice dynamical systems, Nonlinear Anal. 72 (2010) 483-494.

[12] Han X. Random attractors for stochastic sine-Gordon lattice systems with multiplicative white noise, J. Math. Anal. Appl. 376 (2011) 481-493.

[13] Zhao C, Zhou S. Sufficient conditions for the existence of global random attractors for stochastic lattice dynamical systems and applications, J. Math. Anal. Appl. 354 (2009) 78-95.

[14] Han X, Shen W, Zhou S. Random attractors for stochastic lattice dynamical system in weighted spaces, J. Differ. Eqns. 250 (2011) 1235-1266.

[15] Han X. Random attractors for second order stochastic lattice dynamical systems with multiplicative noise in weighted spaces, Stoch. Dyn. 12 (2012) 1150024 1-20.

[16] Han X. Asymptotic behaviors for second order stochastic lattice dynamical systems on $\mathbb{Z}^{k}$ in weighted spaces, J. Math. Anal. Appl. 397 (2013) 242-254.

[17] Maslowski B, Schmalfuß B. Random dynamical systems and stationary solutions of differential equations driven by the fractional Brownian motion, Stochasic Anal. Appl. 22 (2004) 1577-1607.

[18] Garrido-Atienza M. J, Lu K, Schmalfuß B. Random dynamical systems for stochastic equations driven by a fractional Brownian motion, Discrete Contin. Dyn. Syst. 14 (2010) 473493.

[19] Gu A. Random attractors of stochastic lattice dynamical systems driven by fractional Brownian motions, Int. J. Bifurcation Chaos 23 (2013) 1350041 1-9.

[20] Biagini F, Hu Y, Ssendal B, Zhang T. Stochastic Calculus for Fractional Brownian Motion and Applications, Springer-Verlag, London, 2008.

[21] Mishura Y. S, Stochastic Calculus for Fractional Brownian Motion and Related Processes, Springer-Verlag, Berlin, 2008. 
[22] Jones C. Stability of the traveling wave solution of the FitzHugh-Nagumo System, Trans. Amer. Math. Soc. 286 (1984) 431-469.

[23] Elmer C, Van Vleck, E. Spatially discrete FitzHugh-Nagumo equations, SIAM J. Appl. Math. 96(2005) 1153-1174.

[24] Wang Y, Liu Y, Wang Z. Random attractors for partly dissipative stochastic lattice dynamical systems, J. Difference Equa Appl. 14 (2008) 799-817.

[25] Gu A, Zhou S, Jin Q. Random attractor for partly dissipative stochastic lattice dynamical systems with multiplicative white noises, Acta. Math. Appl. Sin-E. (2012), article in press.

[26] Garrido-Atienza M. J, Kloeden P. E, Neuenkirch A. Discretization of stationary solutions of stochastic systems driven by a fractional Brownian motion, Appl. Math. Optim. 60 (2009) $151-172$.

[27] Zahle M. Integration with respect to fractal functions and stochastic calculus, I, Probab. Theory Relat. Fields 111 (1998) 333-374.

[28] Decreusefond L, Üstünel A. S. Stochastic analysis of the fractional Brownian motion, Potential Anal. 10 (1999) 177-214.

[29] Nualart D, Răscanu A. Differential equations driven by fractional Brownian motion, Collect. Math. 53 (2002) 55-81.

[30] Tindel S, Tudor C, Viens F. Stochastic evolution equations with fractional Brownian motion, Probab. Theory Relat. Fields 127 (2003) 186-204.

[31] Arnold L. Random Dynamical Systems, Springer-Verlag, Berlin, 1998.

[32] Chueshov I. Monotone Random Systems Theory and Applications, Springer-Verlag, New York, 2002.

[33] Crauel H, Debussche A, Flandoli F. Random attractors, J. Dyn. Differ. Equat. 9 (1997) 307-341.

[34] Kunita H. Stochastic Flow and Stochasic Differential Equations, Cambridge University Press, Cambridge, 1990.

[35] Garrido-Atienza M. J, Schmalfuß B. Ergodicity of the infinite dimensional fractional Brownian motion, J. Dyn. Differ. Equat. 23 (2011) 671-681. 